\title{
Mitigation of Demagnetization of Bulk Superconductors by Time-Varying External Magnetic Fields
}

\author{
Jin Zou, Student Member, IEEE, Mark D. Ainslie, Member, IEEE, Di Hu, Student Member, IEEE, and David A. \\ Cardwell
}

\begin{abstract}
Large, single-grain high-temperature superconducting (HTS) bulks have significant potential to replace permanent magnets in various engineering applications. However, based on our previous research, the trapped field in a bulk superconductor can be attenuated or even erased when a bulk is subjected to a time-varying, external magnetic field. Therefore, it is important to develop a method to protect bulks from demagnetization by (a) improving the thermal conduction of the bulk and/or (b) reducing AC losses. Improvement in the thermal conduction of bulks involves modification of the material fabrication process, which may have a detrimental effect on its superconducting properties. Employing shielding materials around a bulk helps to decrease the AC losses, but also provides a durable way to maintain the original material properties. In this paper, two shielding cases are proposed and evaluated numerically: ringshaped shielding with a copper coil, and surface shielding with a ferromagnetic material. Based on the numerical modelling results, the ring-shaped coil works well for externally applied AC fields of larger magnitude and higher frequency. However, the ferromagnetic material was preferable for surface shielding for relatively lower fields. Finally, an optimal shield design is presented.
\end{abstract}

Index Terms-High-temperature superconductors, numerical simulation, trapped field magnets, trapped field attenuation, finite element method.

\section{INTRODUCTION}

$\mathrm{L}$ arge, single-grain (RE)BCO high-temperature superconducting (HTS) bulk materials have great potential to trap high magnetic fields of over $17 \mathrm{~T}$ below $30 \mathrm{~K}$ [1], [2] and up to3 $\mathrm{T}$ at $77 \mathrm{~K}$ [3]. More compact and high-performance applications can be realized by using HTS bulks [4-11], but in practical applications these can be exposed to time-varying magnetic field perturbations. Since this phenomenon affects the magnetic field trapped in the bulks to a large extent, the overall efficiency and performance can be limited. Based on previous studies [12-14], the trapped

Manuscript received Aug 2, 2014. M. D. Ainslie would like to acknowledge financial support from a Royal Academy of Engineering Research Fellowship. D. Hu and J. Zou would like to acknowledge financial support from Churchill College, the China Scholarship Council and the Cambridge Commonwealth, European and International Trust. This work was supported in part by a Henan International Cooperation Grant, China: 144300510014.

J. Zou, M. D. Ainslie, D. Hu and D. A. Cardwell are with the Bulk Superconductivity Group, Department of Engineering, University of Cambridge, Cambridge CB2 1PZ, UK (e-mail:jz351@cam.ac.uk, mark.ainslie@eng.cam.ac.uk,dh455@cam.ac.uk,dc135@cam.ac.uk) magnetic field attenuated and was even erased by the application of an external time-varying magnetic field, even though the amplitude was much smaller than the original trapped magnetic field.

Therefore, it is necessary to figure out the exact mechanisms for this demagnetization effect and to develop a method to suppress the attenuation of trapped field. In our previous research [14], the attenuation of trapped field in HTS bulks subjected to an external time-varying field is attributed to flux creep, shielding current redistribution within the HTS bulks, and AC losses generated by the penetration of the external AC field into the bulk. Hence, it is considered that the demagnetization effect can be suppressed by (a) improving the thermal conduction of the bulk or (b) screening the sample from the external field to reduce the losses in the bulks [1617]. In order to improve the thermal properties of the bulk, a bulk impregnated with low melting point metal was developed to suppress demagnetization in an AC field [15]. However, this method may have a detrimental effect on the bulk's superconducting properties. Employing shielding coils around bulks helps to decrease the AC losses [16-17], but also provides a durable way to maintain the material's original properties.

Comparing a bulk sample without any protection, two sets of shields fabricated from copper and ferromagnetic materials are investigated numerically in this paper. The modelling frame work is summarized in Section II, and two different ways of shielding are described in Section III, including the main simulation results.

\section{NUMERICAL SIMULATION}

\section{A. Numerical Simulation Framework}

The electromagnetic properties are simulated using a twodimensional (2D) axisymmetric model based on the $\boldsymbol{H}$ formulation [14], [18-23] and is solved using the Finite Element Method (FEM) implemented in the Partial Differential Equation (PDE) module of COMSOL Multiphysics 4.3a [24]. More detailed model framework can be found in [25].

The HTS bulk sample used in all the models has a diameter of $20 \mathrm{~mm}$ and thickness of $10 \mathrm{~mm}$. The external magnetic field simulation is split into three time domains:

a) $0 \leq \mathrm{t} \leq 8 \mathrm{~s}$ : Zero field cooling (ZFC) with an applied magnetic field, $B_{\max }=2 \mathrm{~T}$. 
b) $8<\mathrm{t} \leq 12 \mathrm{~s}$ : Sinusoidal external magnetic field of magnitude $B_{\text {ex }}$ and frequency $f$ is applied.

c) $12<\mathrm{t} \leq 16 \mathrm{~s}$ : Relaxation time of $4 \mathrm{~s}$.

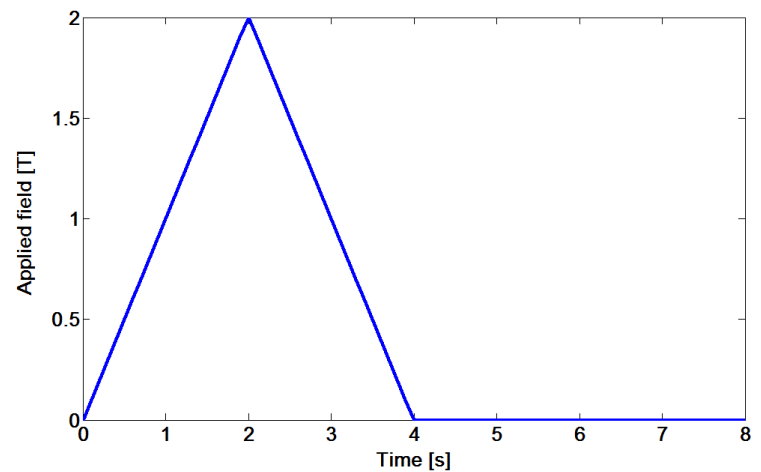

Fig. 1. Applied magnetic field for the simulation of zero field cooling of the YBCO sample during the first stage.

Firstly, the HTS bulk sample is magnetized by the zero field cooling method (ZFC) by setting appropriate boundary conditions in the model to apply the field shown in Fig 1. In this ideal electromagnetic model, any increase in temperature due to the movement of magnetic flux lines is ignored. The reduction of trapped field due to flux creep and shielding current redistribution is analyzed easily in this electromagnetic model without consideration of temperature rise during the process.

The $E-J$ power law $\left(E \alpha J^{\mathrm{n}}\right)$ is used to simulate the nonlinear electrical properties of the superconductors, where $n=$ 21 and $E_{0}=1 \times 10^{-4} \mathrm{~V} / \mathrm{m}$. The Kim model is also employed, as shown in equation (1), to model the $J_{\mathrm{c}}(B)$ characteristics, where $B_{0}=0.4 \mathrm{~T}$ and $J_{\mathrm{c} 0}=2 \times 10^{8} \mathrm{~A} / \mathrm{m}^{2}$, which is consistent with the measured material properties of the (RE)BCO bulk superconductors fabricated by the Cambridge Bulk Superconductivity Group [22].

$$
J_{c}(B)=\frac{J_{c 0}}{\left(1+\frac{B}{B_{0}}\right)}
$$

The governing functions are derived from Maxwell's equations, namely Faraday's (2) and Ampere's (3) laws:

$$
\begin{gathered}
\nabla \times \boldsymbol{E}+\left(\frac{d \boldsymbol{B}}{d t}\right)=\nabla \times \boldsymbol{E}+\frac{d\left(\mu_{0} \mu_{\mathrm{r}} \boldsymbol{H}\right)}{d t}=0 \\
\nabla \times \boldsymbol{H}=\boldsymbol{J}
\end{gathered}
$$

where $\mu_{0}$ is the permeability of free space, and for the superconducting, copper and air sub-domains, the relative permeability is simply $\mu_{\mathrm{r}}=1$. For the ferromagnetic subdomains, an appropriate constant value for $\mu_{\mathrm{r}}$ is used, resulting in a linear $B(H)$ curve, until the saturation value $\mu_{0} M_{\text {sat }}$ is reached. Thus, $\mu_{\mathrm{r}}$ is represented in the numerical simulation by the following equations:

$$
\begin{aligned}
& \mu_{r}=\mu_{r, \max } \text { for } B<\mu_{0} M_{\text {sat }}, \\
& \mu_{r}=\left(1+\frac{M_{\text {sat }}}{H}\right) \text { for } B \geq \mu_{0} M_{\text {sat }}
\end{aligned}
$$

Where $\mu_{0} M_{\text {sat }}$ and $\mu_{\mathrm{r}, \max }$ are $2 \mathrm{~T}$ and 1700 , respectively [21]. In addition, the resistivity of ferromagnetic material used was $1 \times 10^{-6} \Omega \mathrm{m}[21]$.

In these cases, the main focus is the trapped field in the superconductor, and in Section III.C, the loss in the superconductor is considered, but eddy current losses and iron losses have not been considered. A further study that includes a thermal model and the effect of these losses should be investigated in the future, but it is expected that a layer of thermal insulator would be present to isolate the bulk from the shield to avoid any excessive heat transfer to the bulk.

\section{SimULATION RESULTS AND ANALYSIS}

In this section, two types of shield configurations - ringshaped shielding and surface shielding - are investigated and compared with the trapped field performance of the bulk sample without any protection.

\section{A. Ring-shaped Shielding}

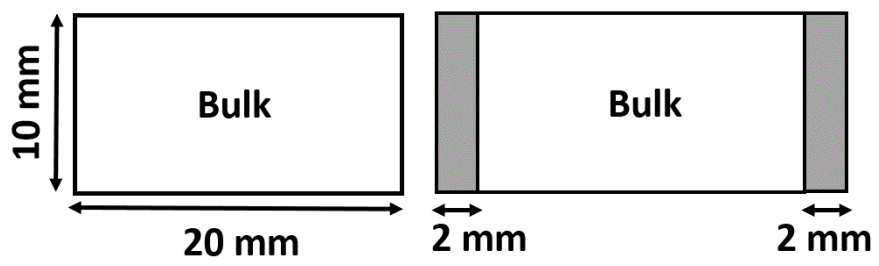

Fig. 2. Bulk sample dimensions (left) and shield configuration made ofa copper or ferromagnetic coil in a ring shape (right).

As shown in Fig. 2, a copper or ferromagnetic shield of thickness $2 \mathrm{~mm}$ was employed to screen the bulk sample from the applied AC field. Due to the effective screening effect, the initial trapped field in the sample with a copper ring $(0.52 \mathrm{~T})$ was slightly lower than that trapped without any shield $(0.54 \mathrm{~T})$. The initial trapped field for the ferromagnetic shielding ring is close to $0.54 \mathrm{~T}$.

As discussed in [14], the trapped field can decrease dramatically with increasing magnitude of external AC field: when the applied field was around $0.3 \mathrm{~T}$, the trapped field was almost erased from the sample. Thus, the shielding effect of the copper and ferromagnetic shields with applied field varying from $B_{\mathrm{ex}}=0.1 \mathrm{~T}$ to $0.3 \mathrm{~T}$ with $f=50 \mathrm{~Hz}$ was investigated using the model and the results are shown in Fig. 3 . With an increase in the applied external field, the remnant trapped field ratio $\left(B_{\text {trap }} / B_{\text {initial }}\right)$ without shielding dropped dramatically from 0.75 to 0.45 . However, with the copper protector, this ratio was controlled within the range of 0.85 to 0.6. The performance of the shield is determined by the resistivity, $\rho$, of the employed copper and also the applied field frequency. In this study, the resistivity of copper was set as 3 $\mathrm{n} \Omega \mathrm{m}$ [26]. In addition, the shielding effect for copper ring is better for relatively higher external applied AC fields, $B_{\text {ex }}$.

However, for the ferromagnetic shielding ring, there is a negligible shielding effect. Due to the field enhancement in ferromagnetic material region during the cycles of the external applied field, the total field around the edge of the bulk sample is higher than without shielding, which on the contrary, even enhances the demagnetization effect. 


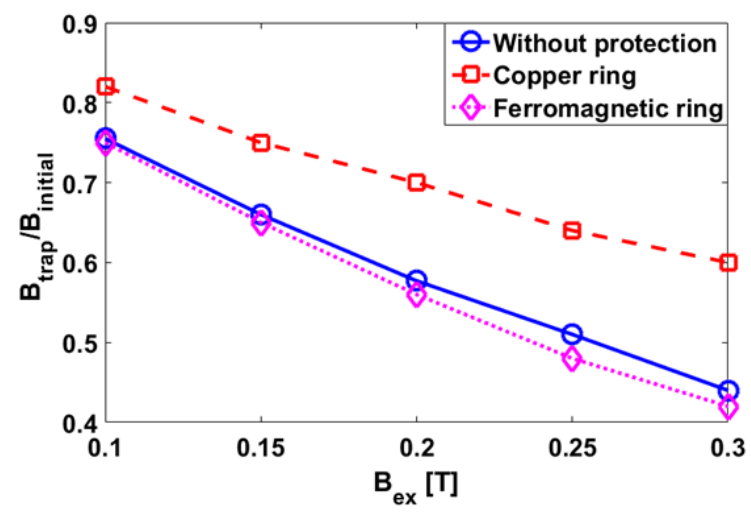

Fig. 3. Remnant trapped field ratio $\left(B_{\text {trap }} / B_{\text {initial }}\right)$ with the applied field magnitude, $B_{\mathrm{ex}}$, varying from 0.1 to $0.3 \mathrm{~T}$, for $f=50 \mathrm{~Hz}$.

The frequency-dependent performance was also assessed. Since the attenuation of the trapped field was more severe for $B_{\mathrm{ex}}=0.3 \mathrm{~T}$ [14], the frequency-dependent performance in the range of 10 to $100 \mathrm{~Hz}$ is analysed for a field, $B_{\text {ex }}=0.3 \mathrm{~T}$, and the results are shown in Fig. 4. Interestingly, with an increase of applied field frequency, the copper shield's performance also improved. This could be explained by Faraday's law. With a faster change in applied field, the induced field generated in the copper coil will be also be relatively large in the opposite direction to compensate the applied AC field, which enhances the shielding effect to protect the trapped field.

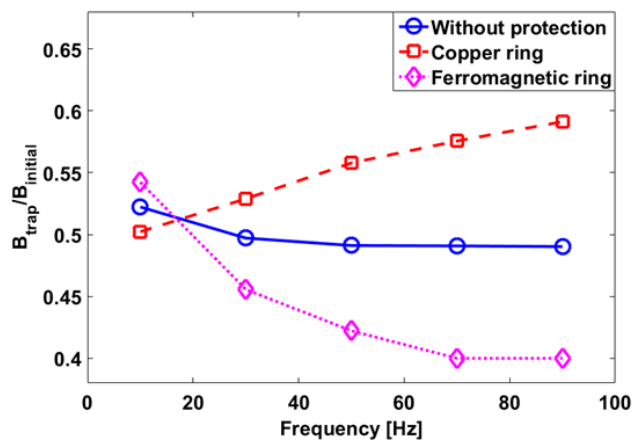

Fig. 4.Frequency dependence of remnant trapped field ratio $\left(B_{\text {trap }} / B_{\text {initial }}\right)$ with the applied field frequency varying from 10 to $90 \mathrm{~Hz}$, for $B_{\mathrm{ex}}=0.3 \mathrm{~T}$.

\section{B. Surface shielding}

Since the use of ferromagnetic materials can enhance the magnetic field in certain regions, another form of shield using ferromagnetic material was investigated, as shown in Fig. 5.

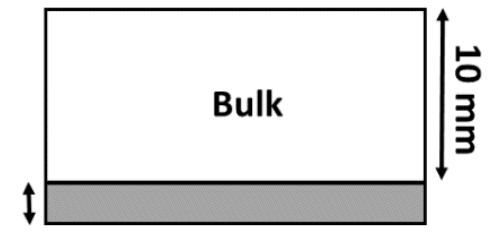

\section{$2 \mathrm{~mm}$}

Fig. 5. Surface shield configuration using ferromagnetic material.

The remnant trapped field ratio when using ferromagnetic surface shielding was studied for $B_{\mathrm{ex}}=0.1$ to $0.3 \mathrm{~T}(f=50 \mathrm{~Hz})$. In Section III.A, the initial trapped field without protection was $0.54 \mathrm{~T}$; however, $B_{\text {initial }}$ in the case of a ferromagnet on the bottom surface is $0.58 \mathrm{~T}$. Thus, the ferromagnetic surface can facilitate the enhancement in trapped field, as shown in [21].

The performance of the ferromagnetic surface shield does not depend on the external AC field frequency, which improves the final trapped field after the application of external time-varying field. However, with a higher magnitude of external field, this mitigation performance was less, and this is shown in Fig. 6.

For a fixed frequency of applied external field $(f=50 \mathrm{~Hz})$, the magnitude-dependence is investigated in Fig. 6. It is shown that the mitigation effect during the applied timevarying field stage (second stage) contributed by the ferromagnetic surface shield is relatively large when $B_{\mathrm{ex}}=0.1$ $\mathrm{T}$, compared with the case where $B_{\mathrm{ex}}=0.3 \mathrm{~T}$.

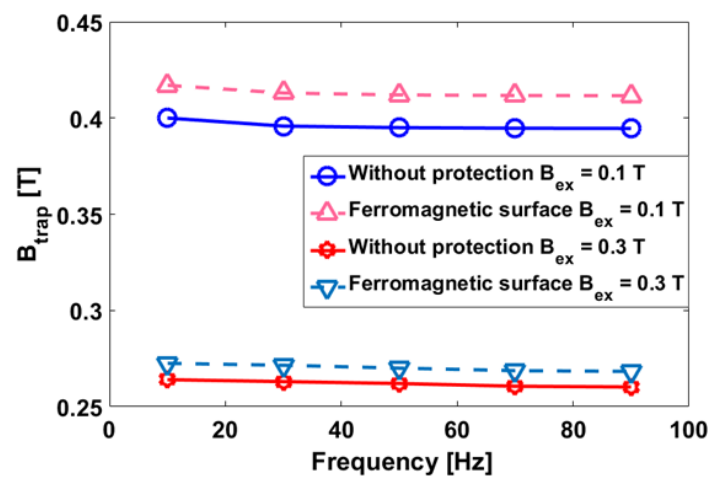

Fig. 6. Frequency dependence of remnant trapped field with the applied field frequency varying from 10 to $90 \mathrm{~Hz}$, for $B_{\mathrm{ex}}=0.1$ and $0.3 \mathrm{~T}$.

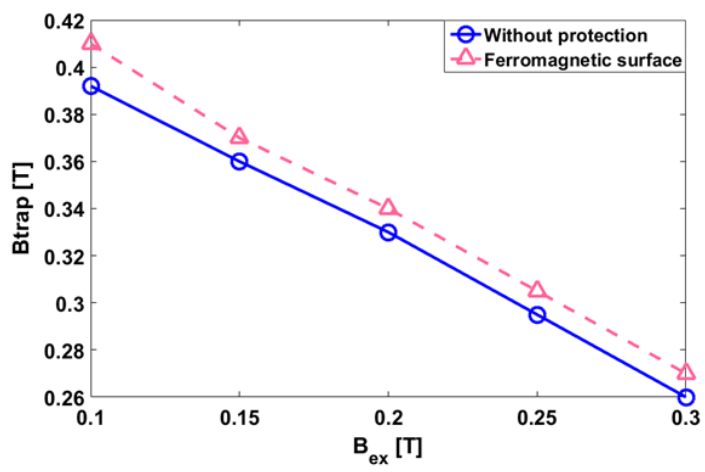

Fig. 7. Remnant trapped field with the applied field magnitude varying from 0.1 to $0.3 \mathrm{~T}$, for $f=50 \mathrm{~Hz}$.

The mitigation performance of the ferromagnetic surface shield is a passive effect, which means if the magnitude of applied field is below the saturation field and the final trapped field near the ferromagnetic surface is relatively high, the enhancement performance will be also high as shown in equation (5):

$$
\boldsymbol{B}=\mu_{0} \mu_{\mathrm{r}} \boldsymbol{H}
$$

where $\mu_{\mathrm{r}}$ is the relative permeability of the ferromagnetic material. As discussed in [14], in the area of penetration of the external applied field, the screening current cannot effectively contribute to the trapped field and reduces the overall trapped field. For a larger external applied AC field, a greater area is 
penetrated by the external field as shown in Figs. 8 and 9 . Therefore, in the case of $B_{\mathrm{ex}}=0.3 \mathrm{~T}$, a larger demagnetization effect occurs and the remnant field around the ferromagnetic surface is relatively smaller than in the case of $B_{\mathrm{ex}}=0.1 \mathrm{~T}$. Thus, the mitigation performance is lower in Fig. 9.

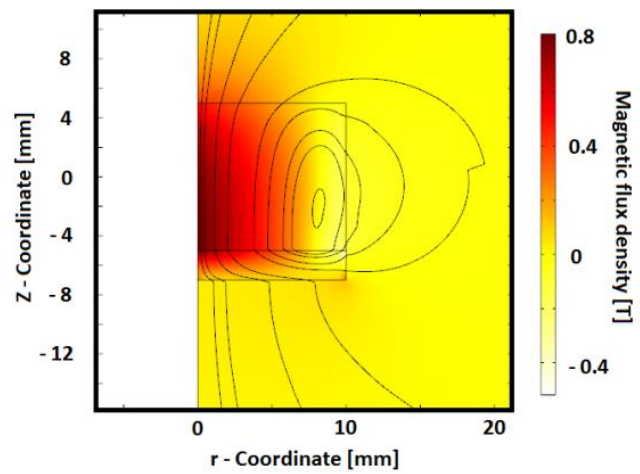

Fig. 8. Magnetic flux density plot of the modulus of B (colour scale) for the surface shielding case with $B_{\mathrm{ex}}=0.1 \mathrm{~T}$ and $f=50 \mathrm{~Hz}$, at $t=16 \mathrm{~s}$.

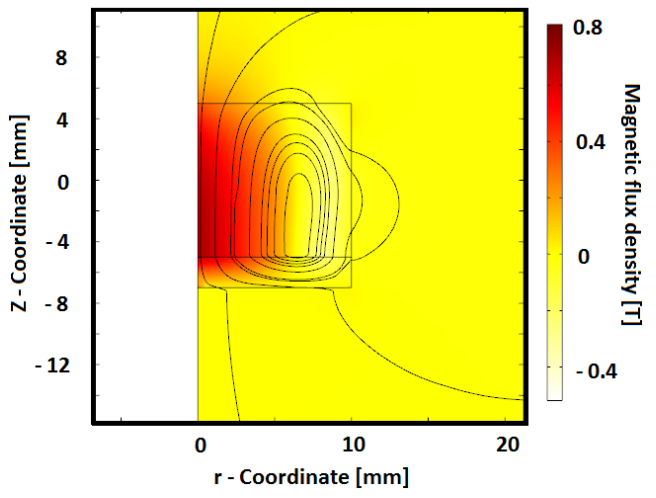

Fig. 9. Magnetic flux density plot of the modulus of B (colour scale) for the surface shielding case with $B_{\mathrm{ex}}=0.3 \mathrm{~T}$ and $f=50 \mathrm{~Hz}$, at $t=16 \mathrm{~s}$.

Fig. 10 shows the remnant trapped field ratio $\left(B_{\text {trap }} / B_{\text {initial }}\right)$ for the ferromagnetic surface shield with the applied field frequency varying from 10 to $90 \mathrm{~Hz}$, for $B_{\mathrm{ex}}=0.3 \mathrm{~T}$. Fig. 11 shows the field-dependent performance of the ferromagnetic surface shield. Although the magnitude of the remnant trapped field for the ferromagnetic surface shield after several cycles of time-varying external applied field was larger than that without protection, the remnant trapped field ratio was worse. The enhancement of remnant trapped field is mainly due to the field enhancement during the ZFC stage and the thickness can be optimized, by finding the threshold thickness for the ferromagnetic surface shield, as described in [21].

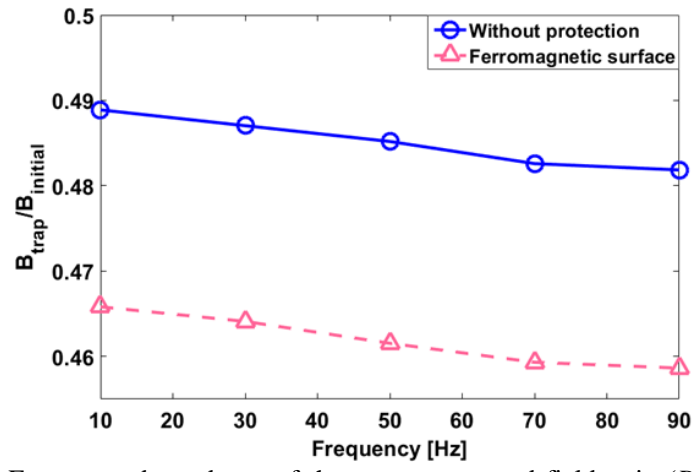

Fig.10. Frequency-dependence of the remnant trapped field ratio $\left(B_{\text {trap }} / B_{\text {initial }}\right)$ with the applied field frequency varying from 10 to $90 \mathrm{~Hz}$, for $B_{\mathrm{ex}}=0.3 \mathrm{~T}$.

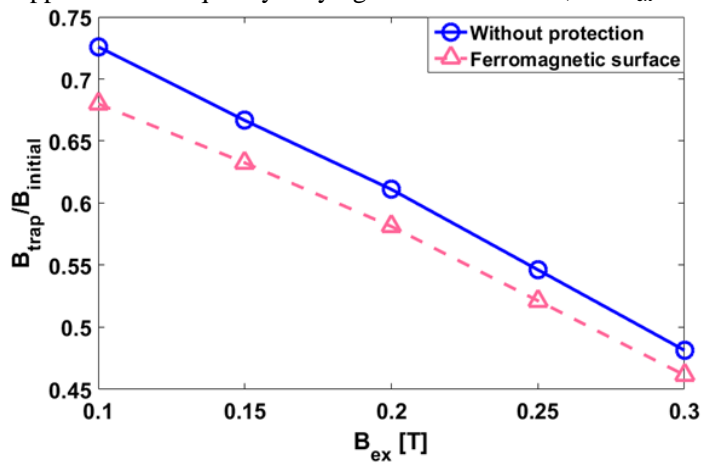

Fig. 11. Remnant trapped field ratio $\left(B_{\text {trap }} / B_{\text {initial }}\right)$ with the applied field magnitude varying from 0.1 to $0.3 \mathrm{~T}$, for $f=50 \mathrm{~Hz}$.

\section{Energy loss in the bulk sample}

Fig. 12 shows 5 cycles of the energy loss ( $Q=\int E \square J d s$ ) for three cases: 1) without protection, 2) with the copper ring, and 3) with ferromagnetic surface shielding. The highest energy loss occurs in the sample without protection. The copper ring shielding reduces the energy loss in the superconductor by approximately one third. Although the energy loss in the bulk sample remains similar in the case of the ferromagnetic surface shield, the remnant trapped field is still slightly higher compared to the case without any protection, due to the enhancement in initial trapped field.

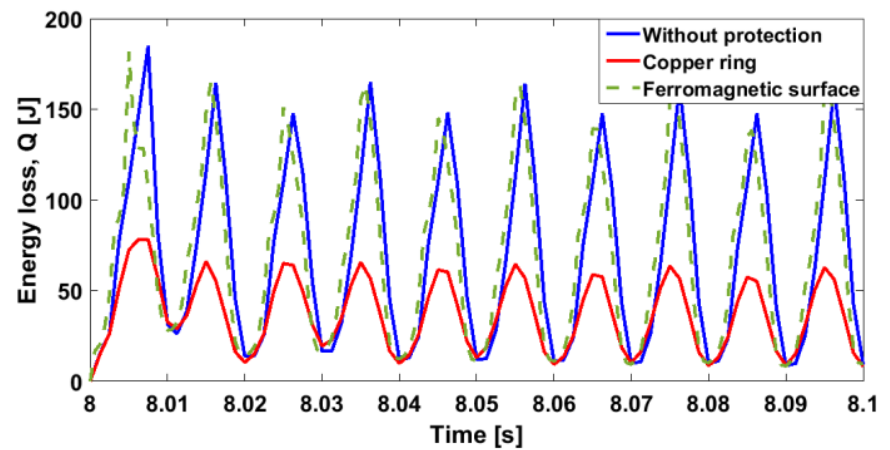

Fig. 12. Instantaneous energy loss in the bulk sample: 1) without protection, 2) with copper ring shielding, and3) with ferromagnetic surface shielding, from $t=11 \mathrm{~s}$ to $11.1 \mathrm{~s}$ for $B_{\mathrm{ex}}=0.2 \mathrm{~T}$ and $f=50 \mathrm{~Hz}$. 


\section{CONCLUSION}

HTS bulk superconductors are ideal candidates to develop more compact and efficient devices, such as actuators, magnetic levitation systems, flywheel energy storage systems and electric machines, due to their ability to trap large magnetic fields. However, a significant concern for practical applications is the attenuation of the trapped field in HTS bulks due to external time-varying fields. In this paper, two types of shielding were studied numerically using copper and ferromagnetic materials.

It is found that copper works well in a ring-shaped shield, particularly for larger external applied fields and higher frequencies, due to the induced shielding current. The ferromagnetic ring-shaped shield has a negligible effect on demagnetization from an external field and can, on the contrary, enhance the demagnetization effect.

The ferromagnetic material was preferable as a surface shield for relatively low external applied fields, but the positive effect on remnant trapped field works due to the field enhancement in the initial magnetizing stage. A threshold thickness can be estimated to optimize the trapped field enhancement from the ferromagnetic surface shield.

\section{REFERENCES}

[1] M. Tomita and M. Murakami, "High-temperature superconductor bulk magnets that can trap magnetic fields of over 17 tesla at $29 \mathrm{~K}$," Nature, vol. 421, no. 6922, pp. 517-520, Jan. 2003.

[2] J. H. Durrell et al., "A trapped field of 17.6 T in melt-processed, bulk Gd-Ba-Cu-O reinforced with shrink-fit steel," Supercond. Sci. Technol., vol. 27, no. 8, Aug. 2014, Art. ID 082001.

[3] S. Nariki, N. Sakai, and M. Murakami, "Melt-processed Gd-Ba-Cu-O superconductor with trapped field of $3 \mathrm{~T}$ at $77 \mathrm{~K}$," Supercond. Sci. Technol., vol. 18, no. 2, pp. S126-S130, Feb. 2005.

[4] B. Oswald et al., "Reluctance motors with bulk HTS material," Supercond. Sci. Technol, vol. 18, no. 2, pp. S24-S29, Feb. 2005.

[5] X. Granados et al., "Low-power superconducting motors," Supercond. Sci. Technol. vol. 21, no. 3, Mar. 2008, Art. ID 034010.

[6] Y. Jiang et al., "The design, magnetization and control of a superconducting permanent magnet synchronous motor," Supercond. Sci. Technol. vol. 21, no. 6, Jun. 2008, Art. ID 065011.

[7] P. J. Masson et al., "Design of HTS axial flux motor for aircraft propulsion," IEEE Trans. Appl. Supercond. vol. 17, no. 2, pp. 15331536, Jun. 2007.

[8] J. R. Hull, "Superconducting bearings," Supercond. Sci. Technol. vol. 13, no. 2, p. R1-R14, Feb. 2000.

[9] H. Sino, K. Nagashima, and Y. Arai, "Development of superconducting magnetic bearing using superconducting coil and bulk superconductor," J. Phys.: Conf. Ser., vol. 97, 2008, Art. ID 012101.

[10] N. Koshizuka, "R\&D of superconducting bearing technologies for flywheel energy storage systems," Physica C, vol. 445-448, pp. 11031108 , Oct. 2006.

[11] F. N. Werfel, "HTS magnetic bearings," Physica C, vol. 372-376, pp. 1482-1486, Aug. 2002.

[12] J. Ogawa, M. Iwamoto, O. Tsukamoto, M. Murakami, and M. Tomita, "Interaction between trapped magnetic field and AC loss in HTS bulk," Physica C, vol. 372-376, no. 3, pp. 1754-1757, Aug. 2002.

[13] J. Ogawa, M. Iwamoto, K. Yamagishi, O. Tsukamoto, M. Murakami, and M. Tomita, "Influence of AC external magnetic field perturbation on trapped magnetic field in HTS bulk," Physica C, vol. 386, pp. 26-30, Apr. 2003.

[14] J. Zou, M. D. Ainslie, D. Hu, and D. A. Cardwell, "Influence of TimeVarying External Magnetic Fields on Trapped Fields in Bulk Superconductors," IEEE Trans. Appl. Supercond, vol. 23, no. 3, Jun. 2015, Art. ID 4900505.

[15] Y. Zushi, I. Asaba, J. Ogawa, K. Yamagishi, O. Tsukamoto, M. Murakami, and M. Tomita, "Study on suppression of decay of trapped magnetic field in HTS bulk subject to AC magnetic field," Physica C, vol. 412-414, no. 1, pp. 708-713, Oct. 2004.

[16] K. Yamagishi, O. Tsukamoto, and J. Ogawa, "Suppression of influence of AC external magnetic field on trapped magnetic flux in the HTS bulk by use of HTS shielding rings," IEEE Trans. Appl. Supercond., vol. 19, no. 3, pp. 3561-3564, June 2009.

[17] K. Yamagishi, O. Tsukamoto, J. Ogawa, and D. Miyagi, "Study of method to suppress decay of trapped magnetic fluxes in the HTS bulk subjected to perturbation of external magnetic field by use shielding coil wound of HTS wire," IEEE Trans. Appl. Supercond., vol. 21, no. 3, pp. 3320-3324, Jun. 2011.

[18] M. Zhang et al., "Experimental and numerical study of a YBCO pancake coil with a magnetic substrate," Supercond. Sci. Technol., vol. 25, no. 12, Dec. 2012, Art. ID 125020.

[19] A. Patel and B. A. Glowacki, "Enhanced trapped field achieved in a superconducting bulk using high thermal conductivity structures following simulated pulsed field magnetization," Supercond. Sci. Technol., vol. 25, no. 12, Dec. 2012, Art. ID 125015.

[20] J. Zou et al., "Numerical modelling and comparison of $\mathrm{MgB}_{2}$ bulks fabricated by HIP and infiltration growth," Supercond. Sci. Technol., vol. 28, no. 7, Jul. 2015, Art. ID 075009.

[21] M. P. Philippe et al., "Influence of soft ferromagnetic sections on the magnetic flux density profile of a large grain, bulk Y-Ba-Cu-O superconductor," Supercond. Sci. Technol., vol. 28, no. 9, Jul. 2015, Art. ID 095008

[22] Z. Xu et al., "Simulation studies on the magnetization of (RE)BCO bulk superconductors using various split-coil arrangements," Supercond. Sci. Technol.,vol. 25, no. 2, Feb. 2012, Art. ID 025016.

[23] M. D. Ainslie et al., "Modelling and comparison of trapped fields in (RE)BCO bulk superconductors for activation using pulsed field magnetization," Supercond. Sci. Technol., vol.27, no. 4, Apr. 2014, Art. ID 065008 .

[24] COMSOL, Inc. [Online]. Available: http://www.comsol.com

[25] M. D. Ainslie and H. Fujishiro, "Modelling of bulk superconductor magnetization," Supercond. Sci. Technol., vol. 28, no. 5, May 2015, Art. ID 053002 .

[26] M. Zhang, K Matsuda, and T. A. Coombs, "New application of temperature-dependent modelling of high temperature superconductors: quench propagation and pulse magnetization," J. Appl. Phys., vol. 112, no. 4, Aug. 2012. 